\title{
Modeling of the mechanobiological adaptation in muscular arteries
}

\author{
Stefan Lindström, Jonas Stålhand and Anders Klarbring \\ Journal Article
}

Tweet

N.B.: When citing this work, cite the original article.

Original Publication:

Stefan Lindström, Jonas Stålhand and Anders Klarbring, Modeling of the mechanobiological adaptation in muscular arteries, European journal of mechanics. A, Solids, 2017. 64, pp.165177.

http://dx.doi.org/10.1016/j.euromechsol.2017.01.011

Copyright: Elsevier

http://www.elsevier.com/

Postprint available at: Linköping University Electronic Press

http://urn.kb.se/resolve?urn=urn:nbn:se:liu:diva-137371

(c)

I.U UNNGDESGS 


\title{
Modeling of the mechanobiological adaptation in muscular arteries
}

\author{
Stefan B. Lindström ${ }^{\mathrm{a}}$, Jonas Stålhand ${ }^{\mathrm{a}}$, Anders Klarbring ${ }^{\mathrm{a}}$
}

${ }^{a}$ Solid Mechanics, Department of Management and Engineering, the Institute of Technology, Linköping University, 58183 Linköping, Sweden

\begin{abstract}
The growth and remodeling of arteries, as controlled by the local stress state and the sensory input from the endothelial cells of the artery wall, is given a novel theoretical framework incorporating the active behavior of vascular smooth muscle. We show that local sensory input maps uniquely to the ratio between a target arterial wall cross-section area corresponding to homeostatic conditions and the current arterial wall area. A growth law is formulated by taking the production rates of individual constituents of the arterial wall to be functions of this target-to-current wall area ratio. We find that a minimum active stress response of vascular smooth muscle is necessary to achieve stable adaptation of the artery wall to dynamic flow conditions. With a sufficient active stress alteration in response to stretch, stable growth toward a homeostatic state can be observed for finite step changes or ramp changes in the transmural pressure or the flow rate.
\end{abstract}

Keywords: arterial adaptation, finite elasticity, growth and remodeling

\section{Introduction}

\subsection{Background and motivation}

Arteries grow and remodel to adapt to changing conditions in the human body, whether these changes are through injury, disease or normal aging. During this growth and remodeling (G\&R) process, the constituents of the artery, including collagen, elastin and vascular smooth muscle (Boron and Boulpaep, 2008, pp. 473-481), display different rates of turnover, where the balance between degradation and production rates is purposefully controlled to maintain functionality of the cardiovascular system. At the level of individual arteries, the arterial wall remodels in response to changes in blood pressure (Matsumoto and Hayashi, 1996; Fridez et al., 2002; Hu et al., 2007a,b) or blood flow rate (Langille and O'Donnell, 1986; Lehman et al., 1991; Langille et al., 1989; Brownlee and Langille, 1991). Instantaneous contraction or dilation is achieved by changes in vascular smooth muscle cell tone, which is combined with matrix remodeling at the longer time-scale of G\&R (Rodbard, 1975; Dajnowiec and Langille, 2007). While a qualitative picture of the G\&R of arteries has been elucidated though experiments and previous modeling efforts, quantitative predictability of the G\&R response to, e.g., surgery, injury or vascular deformities remains elusive. This is likely to preclude many advances in the diagnosis and treatment of vascular diseases. For instance, being able to describe the growth response of the arterial wall of an aneurysm would be highly useful for projecting the evolution of the shape of the aneurysm in mechanobiological models.

In this work, we combine thin-wall tube theory with a finite elasticity mechanical model for multiple constituents that deform together as a constrained mixture (Humphrey and Rajagopal, 2002; Gleason and Humphrey, 2004; Valentín and Humphrey, 2009a; Valentín et al., 2009). These constituents are continually degraded and produced, as modulated by mechanical stimulation. The constrained mixture theory permits for using different natural configurations for different constituents, which is necessary for modeling their continuous turnover. This is in contrast to a theory based on the multiplicative split of the deformation gradient which, in the case of growth, is usually attributed to Skalak et al. (1996). In this approach one single natural configuration is used, instead of one such for each constituent as in the constrained mixture theory. For a normally functioning artery at physiologically constant conditions, G\&R controls the evolution of the artery toward a steady-state, which is referred to as the homeostatic state. The constituents of the artery are deposited at a certain prestretch, which is also their homeostatic stretch. Consequently, each constituent carry a known circumferential stress in the homeostatic state (Satha et al., 2014). We assume that G\&R is controlled locally. By this, we mean that each small through-thickness element of the arterial wall (Fig. 1a) grow and remodel according to the sensory input of that element. It is assumed that this sensory input originates from stress-transduction mechanisms in embedded cells as well as the endothelial cells that line the interior wall of the artery.

A range of novel concepts and ideas are introduced in this work: we formulate an energy density functional for the active stress contribution of smooth muscle, capturing the long-time scale phenotypic switching between the contractile and the synthetic state of smooth muscle (Halayko and Solway, 2001; Albinsson et al., 2014); we consider how the stimuli and the mechanical model can be combined to derive information about the current wall cross-section area and lumen radius of the artery, and thus pinpoint the appropriate direction of growth; we identify the stress measure that appears naturally in the governing equations of the system as a growth stimulus. 


\subsection{Stimuli, geometry and adaptation of arteries}

Changes in the volumetric flow rate $u$ affect the lumen diameter through remodeling and smooth muscle activity in the arterial wall. The smooth muscle dilates or contracts to maintain a constant tangential traction on the endothelial cells of the lining of the vessel wall (Brownlee and Langille, 1991). At the timescales of G\&R, the composition of material within the vessel wall changes in response to sustained changes in $u$ (Kubis et al., 2001). These adaptations essentially follow the physiological principle of minimum work: the metabolic power needed to maintain the blood within the vessel and the power needed to overcome the hydrodynamic resistance of the vessel is minimized at a given volumetric flow rate (Murray, 1926), as modulated by the metabolic cost of the vessel wall (Taber, 1998) and the nonlinear mechanics of the artery (Lindström et al., 2015). We argue herein that this process of arterial adaptation toward a target geometry and composition can be formulated in terms of parameters of geometrical origin. To establish this view, we proceed to demonstrate that sensory input from the cells of the artery wall yield information about the relative difference between the current and target blood vessel geometry, as quantified by the lumen radius and the wall cross-section area.

The tangential traction $\tau_{\mathrm{w}}$ at the interior surface of the vessel wall (Fig. 1b), commonly referred to as the wall shear stress, is sensed by the endothelial cells. For a lumen radius $r_{\mathrm{i}}$ (Fig. 1a), the Hagen-Poiseuille solution for laminar flow through a circular tube, with a Newtonian fluid model for blood, gives the wall shear stress

$$
\tau_{\mathrm{w}}=\frac{4 \eta u}{\pi r_{\mathrm{i}}^{3}},
$$

where $\eta$ is the viscosity of blood. Let $\hat{\bullet}$ indicate quantities in a state of unchanging homeostatic conditions. Assuming that the viscosity is constant, the wall shear stress in a homeostatic state becomes $\hat{\tau}_{\mathrm{w}}=4 \eta u /\left(\pi \hat{r}_{\mathrm{i}}^{3}\right)$, which is divided by Eq. (1) to give

$$
\frac{r_{\mathrm{i}}}{\hat{r}_{\mathrm{i}}}=\left(\frac{\hat{\tau}_{\mathrm{w}}}{\tau_{\mathrm{w}}}\right)^{1 / 3} .
$$

Consequently, if the homeostatic wall shear stress $\hat{\tau}_{\mathrm{w}}$ is known to the G\&R control, then the ratio $r_{\mathrm{i}} / \hat{r}_{\mathrm{i}}$ becomes known as well from sensing the current wall shear stress.

From experiments on dog, cat and rat, $\hat{\tau}_{\mathrm{w}}$ is known to vary between 1.0 and 2.5 Pa through the arterial tree (Kamiya et al., 1984), and similar values, $\hat{\tau}_{\mathrm{w}} \approx 1.5 \mathrm{~Pa}$, are reported for human arteries (Humphrey, 2002). We assume herein that the local value for $\hat{\tau}_{\mathrm{w}}$ is known to G\&R control so that there exists a one-to-one relation between $r_{\mathrm{i}} / \hat{r}_{\mathrm{i}}$ and $\tau_{\mathrm{w}}$.

It has been shown experimentally that blood vessels adapt to changes in the time-average blood pressure by changing the wall thickness and composition to achieve a homeostatic state (Matsumoto and Hayashi, 1996; Hu et al., 2007b). This transmural pressure $p$ across the artery wall is not directly accessible to the G\&R control. Let $\sigma_{\varphi}$ denote the average circumferential stress in the arterial wall (Fig. 1c), let $h$ denote the arterial wall thickness, and $A$ the arterial wall cross-section area. For a tubeshaped, thin-walled artery, the mechanical equilibrium yields $h \sigma_{\varphi}-r_{\mathrm{i}} p=0$, so that

$$
A=2 \pi r_{\mathrm{i}} h=2 \pi \frac{p r_{\mathrm{i}}^{2}}{\sigma_{\varphi}} .
$$

By dividing this Eq. (3) for the homeostatic state, $A=\hat{A}$ and $\sigma_{\varphi}=\hat{\sigma}_{\varphi}$, with the same Eq. for the current state, we obtain

$$
\frac{\hat{A}}{A}=\frac{\sigma_{\varphi}}{\hat{\sigma}_{\varphi}} \frac{\hat{r}_{\mathrm{i}}^{2}}{r_{\mathrm{i}}^{2}}=\frac{\sigma_{\varphi}}{\hat{\sigma}_{\varphi}}\left(\frac{\tau_{\mathrm{w}}}{\hat{\tau}_{\mathrm{w}}}\right)^{2 / 3} .
$$

Equation (4) demonstrates how two local stimuli, the wall shear stress and the circumferential stress, can be exploited by G\&R control to deduce the ratio $\hat{A} / A$, and thus the appropriate direction of growth: an increased rate of production when $\hat{A} / A>1$ and a decreased rate of production when $\hat{A} / A<1$.

A number of different, yet similar, growth laws for individual constituents of the arterial wall have been previously suggested. In these, the production rate of each constituent is assumed to depend on the relative difference between the current and homeostatic circumferential stress (Baek et al., 2006, 2007). Similarly, chemical stimulation is assumed to affect the production rate through the difference to a homeostatic stimulation (Baek et al., 2007). Valentín and Humphrey (2009a) observed that a highly significant contribution to the fluctuations in chemical stimulation comes from the endothelial release of vasodilators and vasoconstrictors in response to changes in $\tau_{\mathrm{w}}$, and thus assumed that the production rate of each constituent $k$ is proportional to a factor

$$
1+K_{\sigma}^{k} \Delta \sigma_{\varphi}-K_{\tau}^{k} \Delta \tau_{\mathrm{w}}
$$

with $\Delta \sigma_{\varphi}=\sigma_{\varphi}-\hat{\sigma}_{\varphi}$ and $\Delta \tau_{\mathrm{w}}=\tau_{\mathrm{w}}-\hat{\tau}_{\mathrm{w}}$. It is common to these previously proposed growth laws that at least one unknown parameter for each stimulus and each constituent is introduced.

Viewing G\&R as a control system, $r_{\mathrm{i}}$ and $A$ takes the role of current state variables, while $\hat{r}_{\mathrm{i}}$ and $\hat{A}$ describes a target state governed by homeostatic conditions. We propose that the ideal direction of growth $\hat{A} / A$ indicated by the stimuli governs the production rate of artery wall materials, and that the growth law of arteries should be formulated using this ratio $\hat{A} / A$. The fact that $A$ is relatively easy to access experimentally using, e.g., ultrasonic imaging techniques available in the clinic makes it feasible to quantify the parameters of such a growth law. By linearizing Eq. (4) at the target (homeostatic) state, it can be understood how $\hat{A} / A$ relates to individual stimuli:

$$
\frac{\hat{A}}{A} \approx 1+\frac{\Delta \sigma_{\varphi}}{\hat{\sigma}_{\varphi}}+\frac{2}{3} \frac{\Delta \tau_{\mathrm{w}}}{\hat{\tau}_{\mathrm{w}}} .
$$

We identify $K_{\sigma}^{k}=\hat{\sigma}_{\varphi}^{-1}$ and $K_{\tau}^{k}=-\frac{2}{3} \hat{\tau}_{\mathrm{w}}^{-1}$ in Eq. (5). This highlights the relation between $\hat{A} / A$ and the production rate in the model of Valentín and Humphrey (2009a), and also shows that the exponents of $\sigma_{\varphi} / \hat{\sigma}_{\varphi}$ and $\tau_{\mathrm{w}} / \hat{\tau}_{\mathrm{w}}$ in Eq. (4) control the impact of different growth stimuli independently. We have left to formulate a mechanical model for an artery undergoing G\&R, and then to express Eq. (4) in the context of this mechanical model. 

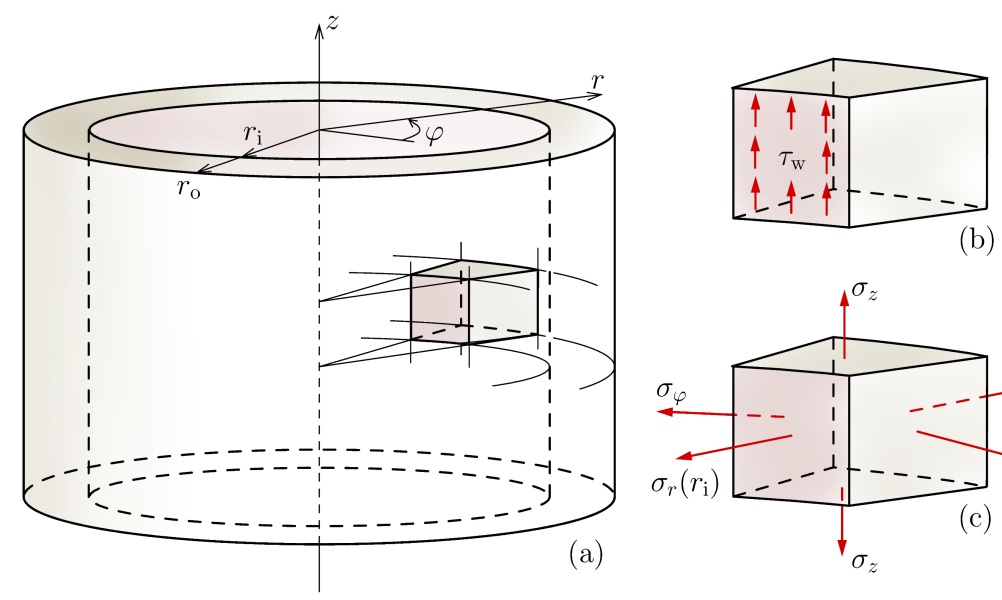

(b)

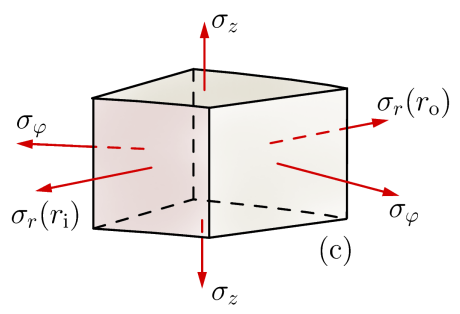

Figure 1: Geometry of the mechanical model of the artery. (a) The tube-shaped artery is aligned with a cylindrical coordinate system. (b) A wall shear stress $\tau_{\mathrm{w}}$ acts on the endothelium of the arterial wall. (c) The principal stresses on a sector of a segment of the arterial wall.

\subsection{Outline}

We start from a thick-walled tube geometry, and formulate a mechanical model using the finite elasticity constrained mixture theory (Humphrey and Rajagopal, 2002; Gleason and Humphrey, 2004; Valentín and Humphrey, 2009a; Valentín et al., 2009; Satha et al., 2014) including an active contribution to stress from the vascular smooth muscle. This together with mechanical equilibrium result in a governing equation (Sect. 2.1). The passive material behavior of each constituent is captured by its strain energy density, which is a functional of the growth history. We demonstrate that the active behavior can be modelled using a similar functional (Sect. 2.2). The ratio $\hat{A} / A$ is then expressed using only the ratio $r_{\mathrm{i}} / \hat{r}_{\mathrm{i}}$ and the stress state of the vessel wall (Sect. 2.4). A growth law is then formulated based on this ratio $\hat{A} / A$ (Sect. 2.5). Numerical simulations are conducted using material parameters from the literature (Sect. 3), and we demonstrate the existence of a domain of stable adaptation to changing flow conditions, including step changes in pressure and volumetric flow rate (Sect. 4).

\section{Theory}

\subsection{Constrained mixture mechanical model}

Constrained mixture theory (Humphrey and Rajagopal, 2002) has been successfully applied to growth of arteries in previous work (Gleason and Humphrey, 2004; Valentín and Humphrey, 2009b; Valentín et al., 2009; Satha et al., 2014; Lindström et al., 2015). In the following section we recapture and extend, by adding active smooth muscle behavior, the derivation of the basic thin-walled equilibrium Eq. (20).

The blood vessel is represented by a thin-walled tube in our mechanical model. However, to be specific about how each assumption enters the theoretical framework, we start our derivation by considering a thick-walled tube, whose reference geometry is parameterized by cylindrical coordinates $(R, \Phi, Z)$ :

$$
R_{\mathrm{i}} \leq R \leq R_{\mathrm{o}}, \quad 0 \leq \Phi \leq 2 \pi, \quad 0 \leq Z \leq L,
$$

where $R_{\mathrm{i}}$ and $R_{\mathrm{O}}$ are the inner and outer reference radii and $L$ is the reference length. A material point $(R, \Phi, Z)$ is mapped onto a new position $(r, \varphi, z)$ in the same coordinate system when deformed (Fig. 1a). This deformation is described as

$$
r=r(R), \quad \varphi=\Phi, \quad z=\kappa Z,
$$

for some function $r(R)$ and parameter $\kappa$. That is, deformations are restricted so that the principal axes of deformation coincide with the coordinate directions with principal stretches

$$
\begin{aligned}
\lambda_{r} & =\frac{\partial r}{\partial R}, \\
\lambda & =\lambda_{\varphi}=\frac{r}{R}, \\
\lambda_{z} & =\kappa .
\end{aligned}
$$

We assume that the blood vessel wall materials preserve their volume when subjected to deformation. This incompressibility is formulated as a constraint, $\lambda_{r} \lambda \lambda_{z}=1$, which gives

$$
\lambda_{r}=\left(\lambda \lambda_{z}\right)^{-1}=\frac{R}{\kappa r} .
$$

The vessel wall comprises a range of different types of biological tissue, with different mechanical properties and growth dynamics. With the constrained mixture theory, all constituents have the same incremental deformation. The constituents are taken to be incompressible, orthotropic materials, with their material directions aligned with axial and cylindrical directions. Each constituent $k$ contributes to the stress through a passive component, as captured by strain energy functions $\psi^{k}$ (Humphrey and Rajagopal, 2002; Gleason and Humphrey, 2004; Valentín and Humphrey, 2009a; Valentín et al., 2009; Satha et al., 2014). These strain energies depend on the material characteristics as well as the growth history of the material. We consider the passive stresses as functions of $\lambda$ and $\lambda_{z}$, with constitutive equations (Ogden, 1997)

$$
\begin{aligned}
\sigma_{\varphi \mathrm{p}}^{k}-\sigma_{r \mathrm{p}}^{k} & =\lambda \frac{\partial \psi^{k}}{\partial \lambda}, \\
\sigma_{z \mathrm{p}}^{k}-\sigma_{r \mathrm{p}}^{k} & =\lambda_{z} \frac{\partial \psi^{k}}{\partial \lambda_{z}},
\end{aligned}
$$


where $\sigma_{r \mathrm{p}}^{k}, \sigma_{\varphi \mathrm{p}}^{k}$ and $\sigma_{z \mathrm{p}}^{k}$ are the passive principal stress components of constituent $k$. In the case of active constituents, i.e. smooth muscle with $k=$ ' $\mathrm{m}$ ', there is also an active contribution to the principal stresses. Since vascular smooth muscle is oriented nearly circumferentially (Rhodin, 1979), their active stress is assumed to be zero in the radial and longitudinal directions, and we postulate that

$$
\sigma_{r \mathrm{a}}^{\mathrm{m}}=0, \quad \sigma_{\varphi \mathrm{a}}^{\mathrm{m}}=\lambda \frac{\partial W}{\partial \lambda}, \quad \sigma_{z \mathrm{a}}^{\mathrm{m}}=0
$$

where we refer to $W$ as the smooth muscle work density. Similarly to the strain energy density, $W$ is a functional of the growth history of the material. This work density $W$ will be given a precise definition and physiological interpretation later by considering the contractile activity of smooth muscle.

The passive and active stresses of smooth muscle are superimposed

$$
\begin{aligned}
\sigma_{r}^{\mathrm{m}} & =\sigma_{r \mathrm{p}}^{\mathrm{m}}+\sigma_{r \mathrm{a}}^{\mathrm{m}}=\sigma_{r \mathrm{p}}^{\mathrm{m}}, \\
\sigma_{\varphi}^{\mathrm{m}} & =\sigma_{\varphi \mathrm{p}}^{\mathrm{m}}+\sigma_{\varphi \mathrm{a}}^{\mathrm{m}}, \\
\sigma_{z}^{\mathrm{m}} & =\sigma_{z \mathrm{p}}^{\mathrm{m}}+\sigma_{z \mathrm{a}}^{\mathrm{m}}=\sigma_{z \mathrm{p}}^{\mathrm{m}},
\end{aligned}
$$

and upon using Eqs. (11a) and (12), we obtain for the active material

$$
\sigma_{\varphi}^{\mathrm{m}}-\sigma_{r}^{\mathrm{m}}=\lambda\left(\frac{\partial \psi^{\mathrm{m}}}{\partial \lambda}+\frac{\partial W}{\partial \lambda}\right)
$$

For all other constituents, $k \neq$ 'm', we have $\sigma_{r}^{k}=\sigma_{r \mathrm{p}}^{k}, \sigma_{\varphi}^{k}=\sigma_{\varphi \mathrm{p}}^{k}$ and $\sigma_{z}^{k}=\sigma_{z \mathrm{p}}^{k}$. The total principal stresses (Fig. 1c) are obtained by applying a mixing rule for the individual constituent principal stresses $\sigma_{r}^{k}, \sigma_{\varphi}^{k}$ and $\sigma_{z}^{k}$ :

$$
\sigma_{\varphi}=\sum_{k} \phi^{k} \sigma_{\varphi}^{k}, \quad \sigma_{r}=\sum_{k} \phi^{k} \sigma_{r}^{k}, \quad \sigma_{z}=\sum_{k} \phi^{k} \sigma_{z}^{k}
$$

where $\phi^{k}$ is the volume fraction of constituent $k$. Note that such a rule-of-mixture is an essential assumption in mixture theory (Humphrey and Rajagopal, 2002) since it enables momentum balance (equilibrium in this work) to be written for the mixture as a whole. A natural extension to this rule for active materials is the superposition of Eqs. (13a)-(13c). However, an alternative to this active stress superposition could possibly be a development based on a multiplicative split of the deformation related to smooth muscles.

The mechanical equilibrium for the radial direction gives

$$
\frac{\partial \sigma_{r}}{\partial r}+\frac{\sigma_{r}-\sigma_{\varphi}}{r}=0
$$

We have $\sigma_{r}=-p_{1}$ at $r_{\mathrm{o}}=r\left(R_{\mathrm{o}}\right)$, with $p_{1}$ the pressure outside the tube, and $\sigma_{r}=-p-p_{1}$ at $r_{\mathrm{i}}=r\left(R_{\mathrm{i}}\right)$, with $p$ the transmural pressure. Integrating Eq. (16) yields

$$
p=\int_{r_{\mathrm{i}}}^{r_{\mathrm{o}}}\left(\sigma_{\varphi}-\sigma_{r}\right) \frac{\mathrm{d} r}{r} .
$$

Substituting Eqs. (11a), (14) and (15) into Eq. (17) then gives

$$
p=\int_{r_{\mathrm{i}}}^{r_{\mathrm{o}}} \lambda \frac{\partial}{\partial \lambda}\left(\sum_{k} \phi^{k} \psi^{k}+\phi^{\mathrm{m}} W\right) \frac{\mathrm{d} r}{r} .
$$

We rewrite the integral of Eq. (18) in terms of the reference coordinate $R$ by observing that $r=\lambda R$ from Eq. (9b), and that $d r=\left(\lambda \lambda_{z}\right)^{-1} d R$ from Eqs. (9a) and (10). In the limit of a thinwalled tube, the variation of the integrand

$$
\frac{1}{\lambda \lambda_{z}} \frac{\partial}{\partial \lambda}\left(\sum_{k} \phi^{k} \psi^{k}+\phi^{\mathrm{m}} W\right)
$$

through the thickness is negligible, and the residual stress (Fung, 1983; Vaishnav and Vossoughi, 1983) is thus neglected. Equation (18) then becomes

$$
p=\frac{1}{2 \pi \lambda \lambda_{z} R_{\mathrm{i}}^{2}} \frac{\partial}{\partial \lambda}\left(\sum_{k} A^{k} \psi^{k}+A^{\mathrm{m}} W\right),
$$

where we define

$$
A^{k}=2 \pi \phi^{k} R_{\mathrm{i}}^{2} \ln \frac{R_{\mathrm{o}}}{R_{\mathrm{i}}}
$$

This quantity $A^{k}$ is referred to as the effective area of constituent $k$. For a thin-walled tube of reference wall thickness $H=R_{\mathrm{o}}-R_{\mathrm{i}} \ll R_{\mathrm{i}}$, we have $A^{k} \approx 2 \pi \phi^{k} R_{\mathrm{i}} H \approx \pi \phi^{k}\left(R_{\mathrm{o}}^{2}-R_{\mathrm{i}}^{2}\right)$. Equation (20) includes two unknown stretches, $\lambda$ and $\lambda_{z}$. In many situations in situ, it holds that $\lambda_{z}=\kappa$ is essentially constant (Takamizawa and Hayashi, 1987; Weizsäcker and Kampp, 1990; Schulze-Bauer et al., 2003). Therefore, we assume herein that the strain energies and the smooth muscle work density are functions of $\lambda$ with $\lambda_{z}$ constant.

\subsection{Evolution of composition and strain energy}

In this section, we are interested in the growth dynamics of the multicomponent living material of a blood vessel. The evolution of the effective areas comprises two terms: The remaining area of the original materials and the remaining area $\mathcal{A}^{k} d \tau$ of the materials formed at time $\tau$. This is formulated as (Baek et al., 2006)

$$
A^{k}=A^{k}(0) Q^{k}(t)+\int_{0}^{t} \mathcal{A}^{k}(\tau) q^{k}(t-\tau) \mathrm{d} \tau, \quad t \geq 0,
$$

where $A^{k}(0)$ is the original effective area of constituent $k, Q^{k}(t)$ is the fraction of constituent $k$ that was produced before time $t=$ 0 and remains at time $t, \mathcal{A}^{k}(t) \geq 0$ is the rate of production of effective area at time $t$, and $q^{k}(t)$ is a monotonically decreasing survival function such that $q(0)=1$.

In a dynamic growth environment, materials produced at different times experience different local stretch. We define $\lambda^{k}(t, \tau)$ as the stretch at time $t$ for materials produced at an earlier time $\tau$ (Baek et al., 2006):

$$
\lambda^{k}(t, \tau)=\frac{\lambda(t)}{\lambda(\tau)} \hat{G}^{k}
$$

The ratio $\lambda(t) / \lambda(\tau)$ is the stretch developed during growth, and $\hat{G}^{k}$ is the prestretch of constituent $k$ which develops at the time of production. In the following, we use the notation $\lambda=\lambda(t)$ for brevity.

We assume that materials created at different time instances contribute to the strain energy in proportion to their remaining 
area fractions. Then, for the passive materials, as well as the passive contribution of smooth muscle, we obtain (Baek et al., 2006)

$$
\begin{aligned}
A^{k} \psi^{k}(\lambda)= & A^{k}(0) Q^{k}(t) \Psi^{k}\left[\lambda^{k}(t, 0)\right]+ \\
& \int_{0}^{t} \mathcal{A}^{k}(\tau) q^{k}(t-\tau) \Psi^{k}\left[\lambda^{k}(t, \tau)\right] \mathrm{d} \tau,
\end{aligned}
$$

where, $\Psi^{k}\left[\lambda^{k}(t, \tau)\right]$ is the strain energy density with respect to a stress-free configuration and characterizes the non-linear, elastic behavior (Baek et al., 2006). By differentiating Eq. (24) with respect to $\lambda$, and multiplying the result by $\lambda$, we obtain an expression for the stress difference $\sigma_{\varphi \mathrm{p}}^{k}-\sigma_{r \mathrm{p}}^{k}, c f$. Eq. (11a), as the sum of stress contributions from remaining area fractions:

$$
\begin{aligned}
A^{k} \lambda \frac{\partial \psi^{k}}{\partial \lambda} & =A^{k}(0) Q^{k}(t) \lambda^{k}(t, 0) d \Psi^{k}\left[\lambda^{k}(t, 0)\right]+ \\
& \int_{0}^{t} \mathcal{A}^{k}(\tau) q^{k}(t-\tau) \lambda^{k}(t, \tau) d \Psi^{k}\left[\lambda^{k}(t, \tau)\right] \mathrm{d} \tau
\end{aligned}
$$

where we use the notation $d f(x)=\mathrm{d} f / \mathrm{d} x$.

\subsection{Active stress and the work density}

The active contribution to the circumferential stress is modeled as $\sigma_{\varphi \mathrm{a}}^{\mathrm{m}}=\lambda^{\mathrm{m}} S$ where $S$ is the generated stress per unit relaxed muscle area. G\&R takes place at a long time-scale where the smooth muscle response is not characterized by a calciumdriven cyclic interaction between the myofilaments. More important is the phenotypic switching between a contractile and a synthetic state (Halayko and Solway, 2001; Albinsson et al., 2014). The amount of organelles for protein and lipid synthesis together with mitochondria are up-regulated in the synthetic state at the expense of contractile apparatus-associated proteins. The smooth muscle cell thereby increases its ability to proliferate, migrate and synthesize extracellular constituents while losing, at least, part of its contractile ability. Phenotypic modulation is controlled by a multitude of environmental cues, but, in particular, the stretch field seems to be an important factor (Owens et al., 2001). We assume herein that phenotypic switching is a continuous process in which the smooth muscle cell gradually gains or loses its contractile response according to the function $S=S(C)$, where $C \geq 0$ is the phenotypic modulation stimulus. It represents the accumulation of stimuli descending from alterations in the stretch field. The generated active stress $S$ is, therefore, associated with the slowly varying tone under normal physiological conditions. This interpretation is in contrast to previous studies where the stimulus of contractility is taken to be the intracellular calcium ion concentration governing the faster muscle contraction response; see, e.g., Rachev and Hayashi (1999) and Humphrey and Wilson (2003). Furthermore, we assume that G\&R organizes the cytoskeletal smooth muscle components such that the operation point remains close to the length which generates maximum muscle force.

Since not all infinitesimal areas of smooth muscle experience the same stretch, we adapt the expression for active stress to a situation with variable $\lambda^{\mathrm{m}}$ according to Eq. (23), so that the total active stress difference $\sigma_{\varphi \mathrm{a}}^{\mathrm{m}}-\sigma_{r \mathrm{a}}^{\mathrm{m}}$ is computed as a sum of active stress difference contributions from remaining infinitesimal area fractions produced at different times, $c f$. Eq. (25):

$$
\begin{aligned}
A^{\mathrm{m}} \lambda \frac{\partial W}{\partial \lambda}= & A^{\mathrm{m}}(0) Q^{\mathrm{m}}(t) \lambda^{\mathrm{m}}(t, 0) S(C)+ \\
& \int_{0}^{t} \mathcal{A}^{\mathrm{m}}(\tau) q^{k}(t-\tau) \lambda^{\mathrm{m}}(t, \tau) S(C) \mathrm{d} \tau,
\end{aligned}
$$

where we used the postulates in Eq. (12) that $\sigma_{\varphi \mathrm{a}}^{\mathrm{m}}=\lambda \partial W / \partial \lambda$ and $\sigma_{r \mathrm{a}}^{\mathrm{m}}=0$ on the left-hand side. The phenotypic modulation of the contractile activity is taken to be

$$
S(C)=S_{\mathrm{M}}\left(1-e^{-C^{2}}\right),
$$

where $S_{\mathrm{M}}$ is the maximum stress capacity of smooth muscle in its fully contractile state.

For a target lumen radius $\hat{r}_{\mathrm{i}}$, the stretch is $\hat{\lambda}=\hat{r}_{\mathrm{i}} / R_{\mathrm{i}}$ and we refer to $\hat{\lambda}$ as the target stretch. From Eq. (2), the ratio $\lambda / \hat{\lambda}=r_{\mathrm{i}} / \hat{r}_{\mathrm{i}}$ is available to $G \& R$ through signaling from endothelial cells. We thus postulate that the time window average phenotypic modulation stimulus $C$ of the vascular smooth muscle depends only on this known ratio $\lambda / \hat{\lambda}$, giving

$$
\begin{aligned}
A^{\mathrm{m}} \lambda \frac{\partial W}{\partial \lambda} & =A^{\mathrm{m}}(0) Q^{\mathrm{m}}(t) \frac{\lambda}{\lambda(0)} \hat{G}^{\mathrm{m}} S\left[C\left(\frac{\lambda}{\hat{\lambda}}\right)\right]+ \\
& \int_{0}^{t} \mathcal{A}^{\mathrm{m}}(\tau) q^{\mathrm{m}}(t-\tau) \frac{\lambda}{\lambda(\tau)} \hat{G}^{\mathrm{m}} S\left[C\left(\frac{\lambda}{\hat{\lambda}}\right)\right] \mathrm{d} \tau .
\end{aligned}
$$

Dividing both sides of Eq. (28) by $\lambda$ and rearranging the factors give

$$
\frac{\partial W}{\partial \lambda}=\frac{\hat{G}^{\mathrm{m}}}{\langle\lambda\rangle^{\mathrm{m}}} S\left[C\left(\frac{\lambda}{\hat{\lambda}}\right)\right]
$$

where the effects of the growth history are captured by a smooth muscle area-weighted harmonic mean of the stretch:

$$
\langle\lambda\rangle^{\mathrm{m}}=A^{\mathrm{m}}\left[\frac{A^{\mathrm{m}}(0)}{\lambda(0)} Q^{\mathrm{m}}(t)+\int_{0}^{t} \frac{\mathcal{A}^{\mathrm{m}}(\tau)}{\lambda(\tau)} q^{\mathrm{m}}(t-\tau) \mathrm{d} \tau\right]^{-1} .
$$

The smooth muscle work density can then be formally defined as

$$
W(\lambda)=\frac{\hat{G}^{\mathrm{m}}}{\langle\lambda\rangle^{\mathrm{m}}} \int_{\hat{\lambda}}^{\lambda} S\left[C\left(\frac{\lambda^{\prime}}{\hat{\lambda}}\right)\right] \mathrm{d} \lambda^{\prime}
$$

where the lower limit of integration is arbitrary owing to the constant term of the primitive function. In a homeostatic state, taking the limit $t \rightarrow \infty$ for Eq. (30) gives $\langle\lambda\rangle^{\mathrm{m}} \equiv \hat{\lambda}$, so that the smooth muscle work density in the homeostatic state becomes

$$
\hat{W}(\lambda)=\frac{\hat{G}^{\mathrm{m}}}{\hat{\lambda}} \int_{\hat{\lambda}}^{\lambda} S\left[C\left(\frac{\lambda^{\prime}}{\hat{\lambda}}\right)\right] \mathrm{d} \lambda^{\prime}
$$

\subsection{Current and target composition of the arterial wall}

There are two classes of constituents:

i. Constituents that degrade, $Q^{k}, q^{k} \rightarrow 0$ as $t \rightarrow \infty$, and grow, $\mathcal{A}^{k} \geq 0$, e.g. collagen and smooth muscle.

ii. Constituents that neither degrade, $Q^{k}=1$, nor grow, $\mathcal{A}^{k}=$ 0, e.g. elastin. 
The set of constituent indices belonging to class (i) and (ii) are denoted by $S_{\mathrm{i}}$ and $S_{\mathrm{ii}}$, respectively.

We assume that, at a given position in the arterial tree, there exists one permissible composition of the arterial wall that yields a homeostatic state represented by target effective areas

$$
\hat{A}^{k}=\hat{A} \hat{\phi}^{k}
$$

where $\hat{A}=\sum_{k} \hat{A}^{k}$ is the total target effective area of the wall. The target area fraction $\hat{\phi}^{k}$ of constituent $k$ varies with position due to the variations of the relative amounts of connective tissue and smooth muscle with position in the vascular system, e.g. smaller arteries have a higher fraction of smooth muscle than larger arteries (Rhodin, 1979).

It has been shown previously that, without any additional assumption, the strain energy density in a homeostatic state is (Satha et al., 2014)

$$
\psi^{k}(\lambda)= \begin{cases}\Psi\left(\frac{\lambda}{\hat{\lambda}} \hat{G}^{k}\right), & k \in S_{\mathrm{i}}, \\ \Psi\left(\frac{\lambda}{\lambda(0)} \hat{G}^{k}\right), & k \in S_{\mathrm{ii}}\end{cases}
$$

For a given set of stationary conditions $(p, \hat{\lambda})$ and $\lambda(0)=\hat{\lambda}$, the equilibrium Eq. (20) in the target (homeostatic) state becomes

$$
2 \pi R_{\mathrm{i}}^{2} \lambda_{z} p \hat{\lambda}=\left.\frac{\partial}{\partial \lambda}\left\{\sum_{k} \hat{A}^{k} \Psi^{k}\left(\frac{\lambda}{\hat{\lambda}} \hat{G}^{k}\right)+\hat{A}^{\mathrm{m}} \hat{W}(\lambda)\right\}\right|_{\lambda=\hat{\lambda}} .
$$

By carrying out the differentiation with respect to $\lambda$ and inserting Eq. (33), we obtain

$$
2 \pi R_{\mathrm{i}}^{2} \lambda_{z} p \hat{\lambda}^{2}=\hat{A}\left[\sum_{k} \hat{\phi}^{k} \hat{G}^{k} d \Psi^{k}\left(\hat{G}^{k}\right)+\hat{\phi}^{\mathrm{m}} \hat{G}^{\mathrm{m}} \hat{S}\right],
$$

which implies that $\hat{A}=\hat{A}\left(p \hat{\lambda}^{2}\right)$. In Eq. (36), $\hat{S}=S[C(1)]$ denotes the generated stress per unit relaxed muscle area in the homeostatic state.

We have formulated a target composition $\hat{A}^{k}$ through Eqs. (33) and (36). To purposefully grow toward the target state, the G\&R control system needs to project known signals to an apparent current state and thus determine the proper direction of growth. Since there is no evidence that the current fractions $\phi^{k}$ of different constituents are known to G\&R control, we assume that the control system employs an approximation that the fraction of each constituent is equal to the target area fraction, $\phi^{k}=\hat{\phi}^{k}$, so that

$$
A^{k}=A \hat{\phi}^{k}
$$

where $A=\sum_{k} A^{k}$ is the total wall area. Inserting these approximate areas into the equilibrium Eq. (20) gives

$$
2 \pi R_{\mathrm{i}}^{2} \lambda_{z} p \lambda^{2}=A\left[\sum_{k} \hat{\phi}^{k} \lambda \frac{\partial \psi^{k}}{\partial \lambda}+\hat{\phi}^{\mathrm{m}} \lambda \frac{\partial W}{\partial \lambda}\right] .
$$

By dividing Eq. (36) with (38) we find that

$$
\frac{\hat{A}}{A}=\frac{s}{\hat{s}} \frac{\hat{\lambda}^{2}}{\lambda^{2}},
$$

where we define

$$
\begin{aligned}
\hat{s} & =\sum_{k} \hat{\phi}^{k} \hat{G}^{k} d \Psi^{k}\left(\hat{G}^{k}\right)+\hat{\phi}^{\mathrm{m}} \hat{G}^{\mathrm{m}} \hat{S}, \\
s & =\sum_{k} \hat{\phi}^{k} \lambda \frac{\partial \psi^{k}}{\partial \lambda}+\hat{\phi}^{\mathrm{m}} \lambda \frac{\partial W}{\partial \lambda} .
\end{aligned}
$$

We assume that the G\&R control system is aware of the wall stress differences $\lambda \partial \psi^{k} / \partial \lambda$ and $\lambda \partial W / \partial \lambda$, cf. Eqs. (11a) and (12). Then, Eq. (39) shows that the ratio between the target effective area and the current effective area is known to the G\&R control system through the known ratio $\lambda / \hat{\lambda}$ and the stress measure $s$ that appears naturally in the equilibrium equation. Equation (39) is essentially the same as Eq. (4) with one subtle difference: the stress $s$ includes an approximation $\phi^{k}=\hat{\phi}^{k}$ to avoid using information that is inaccessible to G\&R control. This crucially permits for using Eq. (39) in the formulation of a growth law.

\subsection{Control of growth and remodeling}

We have made likely that stimuli makes the current-to-target stretch ratio $\lambda / \hat{\lambda}$ available to G\&R through Eq. (2), and that an approximation of the target-to-current effective area ratio $\hat{A} / A$ is available to G\&R through Eq. (39). This means that the G\&R control accesses information about the relative difference between the current and the target arterial wall geometry. The growth of individual constituents then need to be regulated based on this information, so that the vessel remodels into a target (homeostatic) state.

To formulate a growth law for constituents $k \in S_{\mathrm{i}}$, we assume that the rate of production $\mathcal{A}^{k}$ of each constituent is proportional to the number of cells per unit area of the arterial wall, and that this number density, in turn, is proportional to the total wall area, $c f$. Baek et al. (2006):

$$
\mathcal{A}^{k}=A \omega^{k}\left(\frac{\hat{A}}{A}\right)=A \omega^{k}\left(\frac{s}{\hat{s}} \frac{\hat{\lambda}^{2}}{\lambda^{2}}\right), \quad k \in S_{\mathrm{i}},
$$

where $\omega^{k}, k \in S_{\mathrm{i}}$, are rate functions that are to be identified. There is a requirement that the value $\omega^{k}(1)$, corresponding to the target (homeostatic) state, yields the basal growth rate necessary to maintain a target area $\hat{A}^{k}$ for each constituent. Admittedly, Eq. (41) is not the most general form, since $\omega^{k}$ could have an additional dependence on $\lambda / \hat{\lambda}$. Still, our proposed growth law is aligned with a principle that is ubiquitous in control systems: the differential of a quantity is related to the difference (or quotient) between the target and current values of that quantity.

For the following discussion, we assume that constituents degrade according to a Poisson process.

$$
Q^{k}(t)=q^{k}(t)=e^{-v^{k} t}, \quad k \in S_{\mathrm{i}},
$$

where $v^{k}$ is a rate constant capturing the turnover of constituent $k$. In the target state, when $s / \hat{s}=\lambda / \hat{\lambda}=1$ and $\hat{A} / A=1$, substituting Eqs. (41) and (42) into Eq. (22), while taking the 
limit $t \rightarrow \infty$, gives

$$
\begin{aligned}
\hat{A}^{k} & =\lim _{t \rightarrow \infty}\left[A^{k}(0) e^{-v^{k} t}+\int_{0}^{t} \hat{A} \omega^{k}(1) e^{-v^{k}(t-\tau)} \mathrm{d} \tau\right] \\
& =\lim _{t \rightarrow \infty}\left[\hat{A} \omega^{k}(1) \frac{1}{v^{k}}\left(1-e^{-v^{k} t}\right)\right] \\
& =\hat{A} \omega^{k}(1) \frac{1}{v^{k}}, \quad k \in S_{\mathrm{i}} .
\end{aligned}
$$

We then use Eq. (33) to conclude that $\omega^{k}(1)=v^{k} \hat{\phi}^{k}$. To reduce the number of parameters used in the growth law, we linearize $\omega^{k}$ on the right hand side of Eq. (41) around the target state $\hat{A} / A=1$, giving

$$
\mathcal{A}^{k}=A v^{k} \hat{\phi}^{k}\left[1+\beta^{k}\left(\frac{s}{\hat{s}} \frac{\hat{\lambda}^{2}}{\lambda^{2}}-1\right)\right], \quad k \in S_{\mathrm{i}},
$$

where we used Eq. (39), and $\beta^{k}=d \omega^{k}(1) /\left(v^{k} \hat{\phi}^{k}\right)>0$ are unknown coefficients. The stability of this growth law is demonstrated numerically in Sect. 4.

In summary, Eq. (44) governs the evolution of the effective areas $A^{k}(t)$ through Eq. (22). The quantities $\langle\lambda\rangle^{\mathrm{m}}(t), \lambda(t), \psi^{k}(t)$ and $W(t)$ that enter into the right-hand side of Eq. (44) can be calculated directly from the growth history $\mathcal{A}^{k}(\tau), 0 \leq \tau \leq t$ using Eqs. (22), (30), (25), (20) and (31). Since the right-hand side of Eq. (44) depends on the growth history, Eq. (22) is the integral form of a delay differential equation (DDE). An explicit numerical scheme is suggested for the solution of this DDE in Appendix A, and convergence upon decreasing the time-step is demonstrated numerically for this scheme.

\section{Numerical experiments}

The properties of the G\&R equations are investigated numerically, using the parameters of the middle cerebral artery (arteria cerebri media). The constituents of the vessel wall are divided into three families: collagen $(k=$ 'c'), vascular smooth muscle $(k=' \mathrm{~m}$ '), and elastin $(k=' \mathrm{e}$ ').

\subsection{Growth rate parameterization}

An indication of the fraction of smooth muscle to connective tissue for some arteries can be found in Rhodin (1979) but, to the knowledge of the authors, there is no readily available data for variations of the target area fractions $\hat{\phi}^{k}$ of each constituent throughout the whole vascular system. However, for the specific artery under consideration, we use a constant approximation for $\hat{\phi}^{k}$, with $\hat{\phi}^{k}$ the area fraction of constituent $k$ in the middle cerebral artery (Table 1).

\subsection{Material models and parameters}

The principal stretches of each constituent $k$ are $\lambda^{k},\left(\lambda^{k} \lambda_{z}\right)^{-1}$ and $\lambda_{z}$, giving the right stretch Cauchy-Green tensor

$$
\mathbf{C}^{k}=\left[\begin{array}{ccc}
\left(\lambda^{k}\right)^{2} & 0 & 0 \\
0 & \left(\lambda^{k} \lambda_{z}\right)^{-2} & 0 \\
0 & 0 & \lambda_{z}^{2}
\end{array}\right]
$$

Table 1: Material parameters of the middle cerebral artery.

\begin{tabular}{ll}
\hline Material & Properties \\
\hline collagen & $\hat{\phi}^{\mathrm{c}}=0.22^{\dagger}$ \\
& $\hat{G}^{\mathrm{c}}=1.08^{\dagger}$ \\
& $c_{1}=672.5 \mathrm{kPa}^{\dagger}$ \\
& $c_{2}=22^{\dagger}$ \\
& $v^{\mathrm{c}}=1 /(80 \mathrm{day})^{\dagger}$ \\
smooth muscle & $\hat{\phi}^{\mathrm{m}}=0.76^{\dagger}$ \\
& $\hat{G}^{\mathrm{m}}=1.3^{\dagger}$ \\
& $c_{3}=10.0 \mathrm{kPa}$ \\
& $c_{4}=3.5^{\dagger}$ \\
& $v^{\mathrm{m}}=1 /(80 \mathrm{day})^{\dagger}$ \\
& $S_{\mathrm{M}}=100 \mathrm{kPa}$ \\
& $\hat{S}^{\ddagger}=50 \mathrm{kPa}$ \\
& $\hat{\phi}^{\mathrm{e}}=0.02^{\dagger}$ \\
& $\hat{G}^{\mathrm{e}}=1.4^{\dagger}$ \\
& $c_{5}=70.6 \mathrm{kPa}$ \\
\hline
\end{tabular}

${ }^{\dagger}$ Baek et al. (2007). ${ }^{\dagger}$ Rachev and Hayashi (1999).

Following Holzapfel and Ogden (2010), we model the constituents as orthotropic, nonlinear elastic materials. For simplicity, we assume that there is one preferred fiber orientation, which coincides with the circumferential direction. In reality, the number of preferred collagen fiber directions and their orientation vary from point to point in the arterial system (Canham et al., 1991; Finlay et al., 1995; Pandolfi and Holzapfel, 2008; Schriefl et al., 2012). As previously described (Satha et al., 2014; Lindström et al., 2015), the strain energy in Baek et al. (2007) is used for collagen and smooth muscle while elastin is described by a neo-Hookean strain energy as suggested by Holzapfel et al. (2000):

$$
\begin{aligned}
\Psi^{\mathrm{c}} & =\frac{c_{1}}{4 c_{2}}\left\{\exp \left[c_{2}\left(I_{4}^{\mathrm{c}}-1\right)^{2}\right]-1\right\} \\
\Psi^{\mathrm{m}} & =\frac{c_{3}}{4 c_{4}}\left\{\exp \left[c_{4}\left(I_{4}^{\mathrm{m}}-1\right)^{2}\right]-1\right\} \\
\Psi^{\mathrm{e}} & =\frac{c_{5}}{2}\left(I_{0}^{\mathrm{e}}-3\right)
\end{aligned}
$$

where $I_{0}^{k}=\operatorname{tr} \mathbf{C}^{k}$ and $I_{4}^{k}=\left(\lambda^{k}\right)^{2}$ are invariants of the CauchyGreen tensor, while $c_{1}, c_{2}, c_{3}, c_{4}$, and $c_{5}$ are material parameters (Table 1).

Since the phenotypic modulation stimulus $C(\lambda / \hat{\lambda})$ is an unknown function, we represent it using a truncated Taylor expansion around the homeostatic condition, $\lambda / \hat{\lambda}=1$ :

$$
C\left(\frac{\lambda}{\hat{\lambda}}\right)=\hat{C}+C^{\prime}\left(\frac{\lambda}{\hat{\lambda}}-1\right)+O\left[\left(\frac{\lambda}{\hat{\lambda}}-1\right)^{2}\right],
$$

where $\hat{C}=C(1)$ and $C^{\prime}=d C(1)$ are nondimensional constants. Here, $\hat{C}$ is the phenotypic modulation stimulus of smooth muscle under homeostatic conditions that satisfies $S(\hat{C})=\hat{S}$, where $\hat{S}=50 \mathrm{kPa}$ is the homeostatic generated stress per unit relaxed muscle area, identical to the activity of smooth muscle under normal physiological conditions (Rachev and Hayashi, 1999). 
Equation (27) then gives

$$
\hat{C}=\sqrt{-\ln \left(1-\frac{\hat{S}}{S_{\mathrm{M}}}\right)},
$$

with $\hat{C}=0.8326$ for our choice of parameters. With this choice for $C$, the smooth muscle work density of Eq. (31) becomes

$$
\begin{aligned}
W(\lambda) & =S_{\mathrm{M}} \hat{G}^{\mathrm{m}} \frac{\hat{\lambda}}{\langle\lambda\rangle^{\mathrm{m}}}\left(\frac{\lambda}{\hat{\lambda}}-1\right. \\
& \left.-\frac{\sqrt{\pi}}{2 C^{\prime}}\left\{\operatorname{erf}\left[\hat{C}+C^{\prime}\left(\frac{\lambda}{\hat{\lambda}}-1\right)\right]-\operatorname{erf}(\hat{C})\right\}\right),
\end{aligned}
$$

where $\operatorname{erf}(x)=\frac{2}{\sqrt{\pi}} \int_{0}^{x} e^{-t^{2}} \mathrm{~d} t$ is the error function. The effects of the value of $C^{\prime}$ is investigated numerically in later Sect. 4.1.

\subsection{Initial conditions and simulation parameters}

The radius of the middle cerebral artery is typically $r_{0}=$ $1.4 \mathrm{~mm}$ and the basal transmural pressure is $p=p_{0}=12.1 \mathrm{kPa}$ (Lodi and Ursino, 1999). The initial conditions are taken as being homeostatic with radius $r=r_{0}$ and stretch $\lambda=\lambda_{0}=r_{0} / R_{\mathrm{i}}$. The homeostatic stress measure $\hat{s}$ is known from the material model and Eq. (40a), so that the initial effective area can be computed from Eq. (36) as $A_{0}=2 \pi R_{\mathrm{i}}^{2} \lambda_{z} p_{0} \lambda_{0}^{2} / \hat{s}$. The initial effective area of each constituent $k$ is $A_{0}^{k}=\hat{\phi}^{k} A_{0}$.

The numerical scheme proposed in Appendix A is used for integrating the evolution equation from $t=0$ to $t=100 / v^{\mathrm{c}}$ corresponding to a duration of 8000 days. A time-step $\Delta t=$ $1 /\left(80 v^{c}\right)$ is used for all simulations.

\section{Results and discussion}

While the mechanical properties of the constituents have been specified in the previous Sect. 3, the parameters $\beta^{\mathrm{c}}$ and $\beta^{\mathrm{m}}$ controlling the production rate in Eq. (44), and the slope $C^{\prime}$ of the phenotypic modulation stimulus are unknown.

\subsection{GER Rtability conditional on growth law parameters}

First, we consider the G\&R in response to a step increment in the target stretch, for $C^{\prime}=10$, and for equal coefficients $\beta=\beta^{\mathrm{c}}=\beta^{\mathrm{m}}$ with different values $\beta=\{0.1,1,10\}$. Physiologically, this step increment corresponds to a sudden increase in the volumetric flow rate. When $t<0$ the vessel is maintained in an initial state at a stretch $\lambda_{0}$ and total wall area $A_{0}=\sum_{k} A_{0}^{k}$ (Sect. 3.3). At $t=0$, the target stretch is set to $\hat{\lambda}=1.05 \lambda_{0}$, corresponding to an increase in the blood flow rate, and the state of the vessel is integrated numerically to obtain the development of $\lambda$ and $A=\sum_{k} A^{k}$ (Fig. 2). Stable growth toward the target state is observed when $\beta=1$ and $\beta=10$, but when $\beta=0.1$, neither the target wall area nor the target stretch is reached (Fig. 2). A larger value of $\beta$ results in a more rapid adaptation of the effective area to a step change in the target stretch $\hat{\lambda}$ (Fig. 2b), but the rate at which the stretch adapts to a step change in $\hat{\lambda}$ is essentially independent of the coefficient $\beta$ as long as $\beta \gtrsim 1$ (Fig. 2a). This observation holds for a range of values of $C^{\prime}$ (not shown).
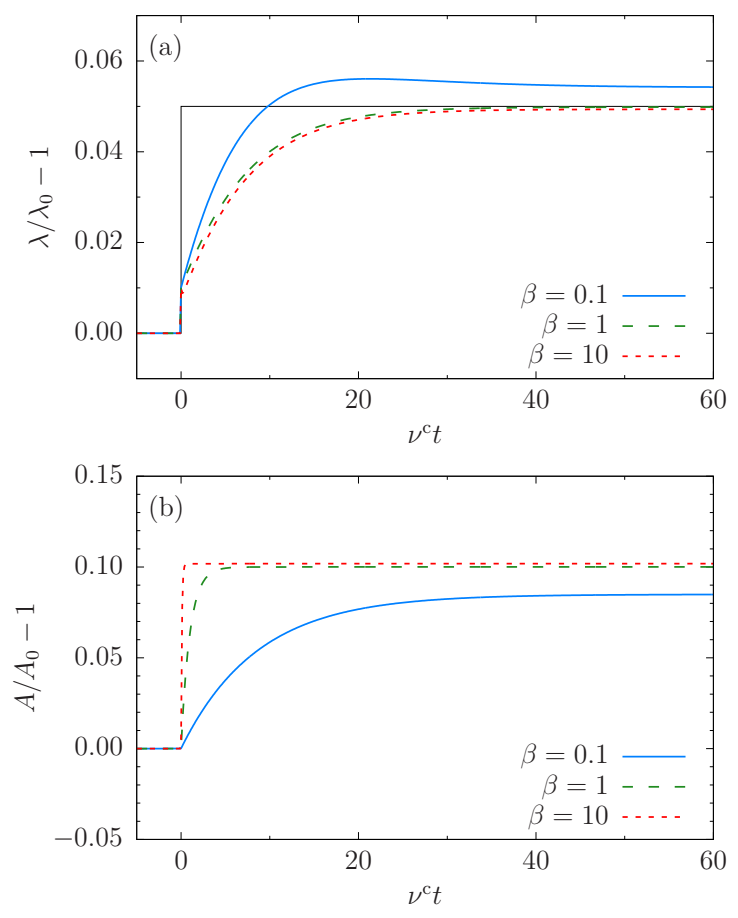

Figure 2: Response to a $5 \%$ step increase in $\hat{\lambda}$ for $C^{\prime}=10$ and for equal coefficients $\beta=\beta^{\mathrm{c}}=\beta^{\mathrm{m}}$. (a) Development of stretch $\lambda$ relative to the initial stretch $\lambda_{0}$. The thin solid line represents the target stretch. (b) Development of vessel wall area $A$ relative to the initial wall area $A_{0}$.
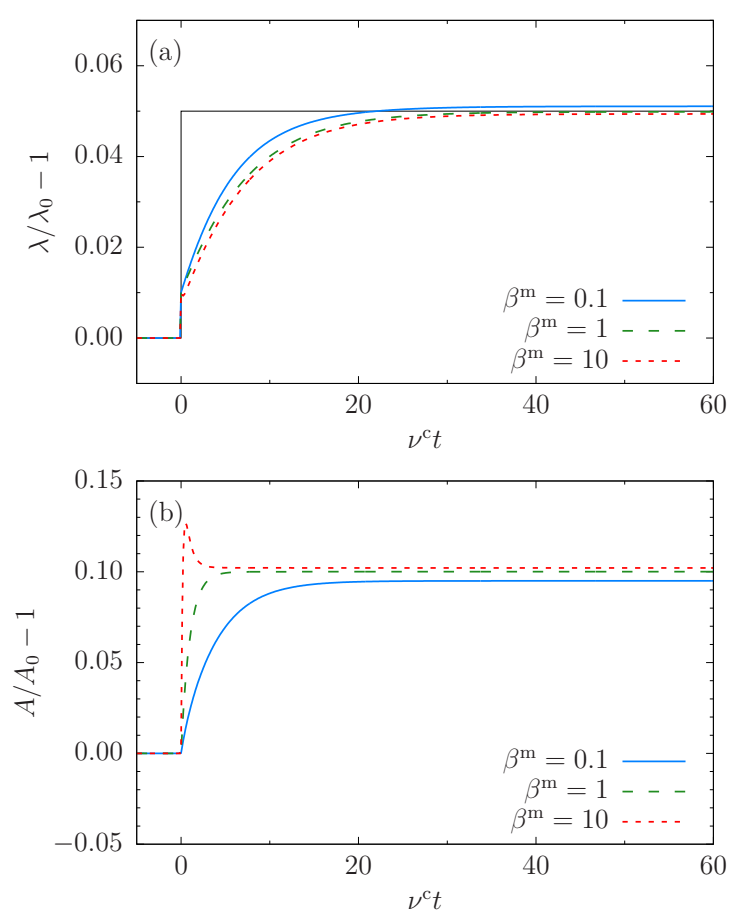

Figure 3: Response to a $5 \%$ step increase in $\hat{\lambda}$ for $C^{\prime}=10, \beta^{\mathrm{c}}=1$ and for different values of $\beta^{\mathrm{m}}$. (a) Development of stretch $\lambda$ relative to the initial stretch $\lambda_{0}$. The thin solid line represents the target stretch. (b) Development of vessel wall area $A$ relative to the initial wall area $A_{0}$. 


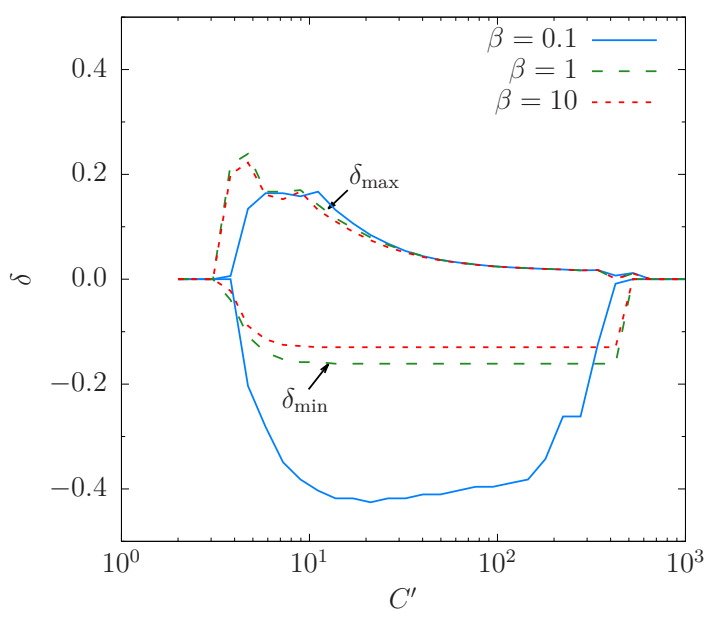

Figure 4: Domain of stable adaptation $\delta_{\min }<\delta<\delta_{\max }$ to a positive or negative relative change $\delta$ in the target stretch for equal coefficients $\beta=\beta^{\mathrm{c}}=\beta^{\mathrm{m}}$.

We investigate the effect of differing rate coefficients by setting $\beta^{\mathrm{c}}=1$ while varying $\beta^{\mathrm{m}}=\{0.1,1,10\}$. Again, we consider the growth response to a $5 \%$ step increase in the target stretch. For $\beta^{\mathrm{m}}=0.1$, neither the target stretch nor the target area is reached (Fig. 3). The development of the stretch is similar for $\beta^{\mathrm{m}}=1$ and $\beta^{\mathrm{m}}=10$ (Fig. 3a). However, the development of the total wall area shows an overshoot for $\beta^{\mathrm{m}}=10$ (Fig. 3b). Consequently, the metabolic energy consumed during adaptation would be larger if $\beta^{\mathrm{m}} \gg \beta^{\mathrm{c}}$ than if $\beta^{\mathrm{m}} \lesssim \beta^{\mathrm{c}}$. The principle of minimum work then suggests that $\beta^{\mathrm{m}} \lesssim \beta^{\mathrm{c}}$. An additional parametric study with $\beta^{\mathrm{m}}=1$ and $\beta^{\mathrm{c}}=\{0.1,1,10\}$ reveals that the effects of changing $\beta^{\mathrm{c}}$ on the stretch and wall area development are small (not shown), likely because the area fraction of collagen is smaller than that of smooth muscle in the investigated case. In the simulations discussed below, we only consider cases with $\beta^{\mathrm{c}}=\beta^{\mathrm{m}}$.

The phenotypic modulation stimulus of smooth muscle is characterized by $\hat{C}$ and $C^{\prime}$, of which the basal stimulus $\hat{C}$ is a known parameter. If $C^{\prime}=0$, the smooth muscle behaves as a passive material in the sense that its active stress becomes insensitive to the current-to-target stretch ratio. It is thus of great interest to investigate how $C^{\prime}$ affects the adaptation of blood vessels to changing conditions.

To investigate the stability of G\&R, the development of the stretch and total effective area in response to different step change in the target stretch, $\delta=\hat{\lambda} / \lambda_{0}-1$, is investigated for different values of $C^{\prime}$. We consider the numerical simulation of G\&R to be stable if

$$
\left|\frac{\lambda\left(t=100 / v^{\mathrm{c}}\right)}{\hat{\lambda}}-1\right| \leq|\delta|
$$

and unstable otherwise. This condition also captures cases when the effective area drifts away from its target value, since such uncontrolled off-drift ultimately affects the development of the stretch. For each value of $C^{\prime}$, there is a, possibly empty, interval $\delta_{\min }<\delta<\delta_{\max }$ within which the G\&R is stable. The bisection method is employed to find $\delta_{\min }\left(C^{\prime}\right)$ and $\delta_{\max }\left(C^{\prime}\right)$, respectively.

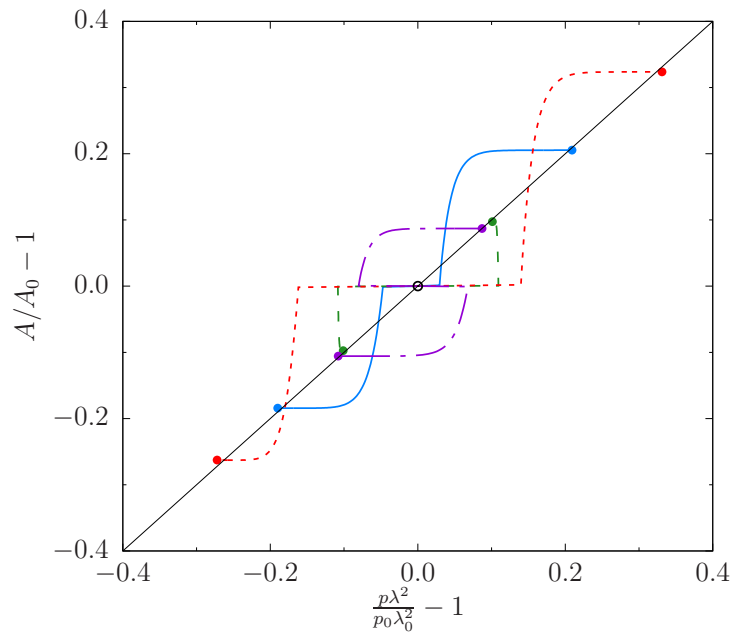

Figure 5: Evolutions in response to $10 \%$ step changes in target stretch or pressure for $\beta^{\mathrm{c}}=\beta^{\mathrm{m}}=1$ and $C^{\prime}=10: \pm 10 \%$ stretch change (thick solid line), $\pm 10 \%$ pressure change (dashed line), $\pm 10 \%$ stretch change and $\pm 10 \%$ pressure change (dotted line), $\pm 10 \%$ stretch change and $\mp 10 \%$ pressure change (dash-dotted line). The initial state of all evolutions is represented by an open circle, while the final states are represented by filled circles. The thin solid line represents homeostatic states.

The domain of stable adaptation is illustrated in Fig. 4 for $\beta^{\mathrm{c}}=\beta^{\mathrm{m}}=1$. This domain ranges from $C^{\prime} \approx 3$ to $C^{\prime} \approx 400$. The existence of a lower limit for $C^{\prime}$ implies that stable G\&R requires a certain minimum active stress alteration with stretch. It is also interesting that stable adaptation is easier to achieve when the target stretch decreases, $\delta<0$, than when it increases, $\delta>0$ (Fig. 4). The ability to adapt to an increasing target stretch is gradually lost as $C^{\prime}$ increases. A wide range of $\delta$ with stable adaptation is obtained when $7 \leq C^{\prime} \leq 15$, in which case $-0.15 \leq \delta \leq 0.1$. For a smaller value of $\beta^{\mathrm{c}}=\beta^{\mathrm{m}}=0.1$, the domain of stable adaptation increases, but the artery does not approach a homeostatic state (Fig. 2). For a larger value of $\beta^{\mathrm{c}}=\beta^{\mathrm{m}}=10$, the domain of stable adaptation becomes smaller. These observations indicate that efficient and stable G\&R control is achieved for parameter values in a neighborhood of $\beta^{\mathrm{c}}=\beta^{\mathrm{m}}=1$ and $C^{\prime}=10$. These parameter values are used in subsequent simulations, unless stated otherwise.

\subsection{GESR under dynamic conditions}

G\&R needs to adapt to changes in the target stretch as well as the pressure. We integrate the evolution of the wall area $A$ and stretch $\lambda$ in response to $10 \%$ step changes in target stretch or pressure, including simultaneous changes in target stretch and pressure (Fig. 5). All these evolutions are stable. As a first response, the smooth muscle always contracts or dilates due to the changing conditions. At a longer time-scale, the wall area and stretch evolve into one of the homeostatic states defined by Eq. (36), with a small error due to the unchanging amount of elastin. The homeostatic states describe the identity function when plotting $A / A_{0}-1$ against $p \lambda^{2} /\left(p_{0} \lambda_{0}^{2}\right)-1$ (Fig. 5).

As demonstrated above (Fig. 4), it is difficult for G\&R to adapt to large step changes in the target stretch, at least for the parameters used herein. This does not necessarily mean that it is 

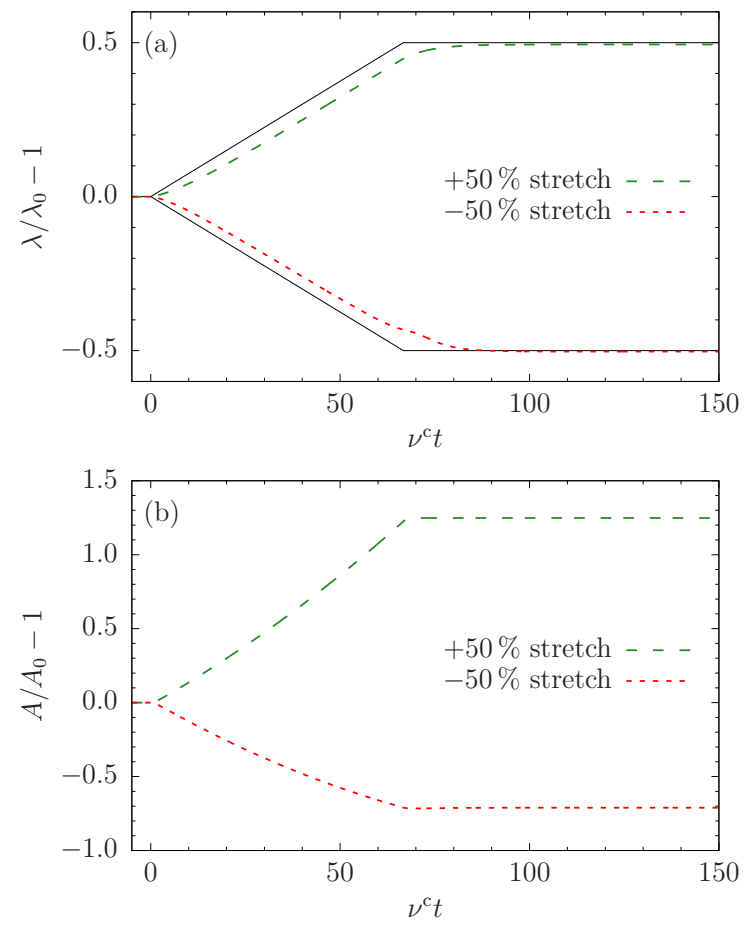

Figure 6: Evolutions in response to ramp changes in the target stretch (solid lines) for $\beta^{\mathrm{c}}=\beta^{\mathrm{m}}=1$ and $C^{\prime}=10$. (a) Development of stretch $\lambda$ relative to the initial stretch $\lambda_{0}$. (b) Development of effective area $A$ relative to the initial area $A_{0}$.

impossible to adapt to large gradual changes. We simulate ramp changes in the target stretch to show that the proposed growth law allows for adaptation to a wide range of conditions (Fig. 6). The plotted cases correspond to the highest ramp rates possible while still maintaining stable adaptation for $\pm 50 \%$ changes in the target stretch.

\subsection{Limitations}

As a final point of this discussion, we summarize the most important model limitations and simplifications.

Arterial walls have a finite thickness and layered structure which includes the adventitia, media and intima (Rhodin, 1979). For such structures, the stress field and the chemical potential become dependent on the radial position and may exhibit discontinuities. In our model, the artery wall is homogenized through the thin-wall assumption, meaning that radial gradients are neglected.

Vascular smooth muscle cells need not be oriented in the axial-circumferential plane. Using electron microscopy imaging, Fujiwara and Uehara (1992) and O'Connel et al. (2008) have shown that the smooth muscle appear at a small radial tilt. As a consequence, torsion will develop in the artery. To the best of the authors' knowledge, there is no indication or quantification in the literature as to the extent of this influence. We therefore limit this study to shear-free deformations.

The smooth muscle normal tone due to the evolution is assumed to be a function of the phenotypic modulation stimulus $C$. This variable is in turn a function of the circumferential stretch through Eq. (47). Previous studies, see, e.g., Zulliger et al. (2002) and Zulliger et al. (2004), have shown that the normal tone of vascular smooth muscle is also affected by the axial stretch. This is not accounted for in the G\&R response since $\lambda_{z}$ is prescribed to a fixed value. Furthermore, Gleason et al. (2007) found that the axial arterial stress in mice also tends toward a homeostatic value. Given that the axial stretch in human arteries decrease with age (Schulze-Bauer et al., 2003; Kamenskiy et al., 2015; Horný et al., 2016), these facts indicate that G\&R should ideally account for alterations in both $\lambda$ and $\lambda_{z}$.

The degradation of the extracellular matrix is assumed to depend only on the rate constant $v^{k}$ in Eq. (42). Experiments have shown that the degradation rate also depends on stress, e.g., matrix-metalloproteinase-driven collagen degradation (Humphrey, 2002, pp. 511-515). It is also possible that the degradation rates $v^{k}$ and the production rate parameters $\beta^{k}$ depend on the current composition of the artery wall. Dependencies of this kind create additional couplings and would increase the complexity of the model. Since this level of detail is felt to be outside the scope of the study, we choose to limit the model to degradation processes governed solely by Eq. (42) and to constant production rate parameters.

Vascular collagen has a half-life of about 15-90 days. This is in sharp contrast to elastin which degrades at a very slow rate, manifested by a half-life on the order of decades (Lefevre and Rucker, 1980). It is therefore assumed that elastin does not degrade within the time-span of our numerical simulations. Note well that this is not a limitation to the model; elastin degradation is straight-forward to include by assigning an appropriate value to $v^{\mathrm{e}}$.

\section{Conclusions}

A growth law describing the evolution of the constituents of the arterial wall is formulated in the context of thin-walled tube theory, constrained mixture theory, finite elasticity and a novel theoretical frame that accounts for the long time-scale active behavior of smooth muscle.

The constitutive relations of passive materials are captured using growth-dependent strain energy densities $\psi^{k}$. At the timescales of G\&R, we show that the active behavior of smooth muscle can be described using a work density function $W$, that takes the role of a strain energy density for active constituents. In contrast to the strain energy density of passive constituents, this work density of smooth muscle depends on the ratio $\lambda / \hat{\lambda}$ between the current stretch and the target stretch corresponding to homeostatic conditions. This current-to-target stretch ratio is available to G\&R control through the wall shear stress that is sensed by endothelial cells.

From the equations of mechanical equilibrium in the homeostatic state and in the current state, respectively, it is possible to estimate the ratio $\hat{A} / A$ between the target and the current wall cross-section area using only the stress state of the vessel wall and the known ratio $\lambda / \hat{\lambda}$. Since $\hat{A} / A$ indicates the appropriate direction of growth for the total wall area, this ratio is assumed to control the production rate of each constituent. As a consequence, only one unknown parameter is needed for each con- 
stituent in the growth law. Since $A$ is experimentally accessible, it is feasible to quantify the parameters, $\beta^{\mathrm{c}}, \beta^{\mathrm{m}}$ and $C^{\prime}$, using a fitting procedure. This is a great advantage as compared to previously suggested models in the literature that, for each constituent, require one model parameter for each type of stimulus (Baek et al., 2007; Valentín and Humphrey, 2009a), and that are based on quantities that are difficult to measure in vivo.

The so-formulated growth law yields stable growth toward a target (homeostatic) state for a range of model parameters. Crucially, it is not possible to achieve stable adaptation to dynamic conditions without a certain minimum active stress alteration with stretch. We conclude that the active response of smooth muscle is necessary to avoid monotonic drift of the lumen radius or the wall area.

Ours formulating the laws governing G\&R for a tube-shaped artery does not restrict its use to this geometry. Since the growth law is formulated using local sensory input, it can be used in an arbitrary geometry. An element of this arbitrary geometry then acts upon its stress state and signals from nearby endothelial cells, as well as the delusion that it is part of a tube-shaped artery. We believe that this is an accurate depiction of reality, since the growth law is encoded in the genome and is thus required to presume a shape and composition for the artery, there being no possibility to detect vascular malformation.

\section{Acknowledgements}

We gratefully acknowledge financial support from the Swedish Research Council under contract Dnr: 621-2012-3117.

\section{Appendix A. Numerical scheme}

An explicit numerical scheme for integrating Eq. (22) is described in detail in this section. Due to history-dependence, the vector of state has infinite dimensionality. Here, we introduce the distribution of remaining areas to represent the growth history.

\section{Appendix A.1. Distribution of remaining areas}

Following Satha et al. (2014), we introduce the distribution of remaining areas

$$
\alpha^{k}(t, \tau)=\mathcal{A}^{k}(\tau) q^{k}(t-\tau), \quad t \geq 0, \quad 0 \leq \tau \leq t,
$$

so that $\alpha^{k}(t, \tau) d \tau$ represents the amount of constituent $k$ that remains at time $t$, and was produced within the time interval $[\tau, \tau+d \tau)$. It follows that

$$
\alpha^{k}(t+\Delta t, \tau)=\frac{q^{k}(t+\Delta t-\tau)}{q^{k}(t-\tau)} \alpha^{k}(t, \tau)
$$

for any constant time-step $\Delta t$, and that

$$
\alpha^{k}(t, t)=\mathcal{A}^{k}(t) q^{k}(0) \quad \Leftrightarrow \quad \mathcal{A}^{k}(t)=\alpha^{k}(t, t) .
$$

We discretize time using a time-step $\Delta t$, with discretization points

$$
t_{n}=n \Delta t, \quad n=0,1,2, \ldots, N
$$

Then, $\alpha^{k}$ becomes discretized as

$$
\alpha_{n, j}^{k}=\alpha^{k}(n \Delta t, j \Delta t), \quad j=0, \ldots, n .
$$

By employing Eq. (A.2), we also have

$$
\alpha_{n+1, j}^{k}=b_{n, j}^{k} \alpha_{n, j}^{k}, \quad b_{n, j}^{k}=\frac{q^{k}[(n-j+1) \Delta t]}{q^{k}[(n-j) \Delta t]} .
$$

\section{Appendix A.2. Time increment iteration}

We propose an explicit numerical scheme to integrate the evolution of the effective areas $A^{k}(t)$ using Eq. (22) with Eq. (44). Slowly-varying quantities, such as $A^{k}$ and $\langle\lambda\rangle^{\mathrm{m}}$, are treated explicitly, while $\lambda$, that changes instantaneously due to changes in the controlling parameters $\hat{\lambda}(t)$ and $p(t)$, is treated implicitly. As a preparation, we divide Eq. (25) by $\lambda$ to obtain

$$
\begin{array}{r}
\frac{\partial}{\partial \lambda}\left(A^{k} \psi^{k}\right)=A^{k}(0) Q^{k}(t) \frac{\hat{G}^{k}}{\lambda(0)} d \Psi^{k}\left[\frac{\lambda}{\lambda(0)} \hat{G}^{k}\right]+ \\
\int_{0}^{t} \mathcal{A}^{k}(\tau) q^{k}(t-\tau) \frac{\hat{G}^{k}}{\lambda(\tau)} d \Psi^{k}\left[\frac{\lambda}{\lambda(\tau)} \hat{G}^{k}\right] \mathrm{d} \tau,
\end{array}
$$

for all $k$. This Eq. (A.7) describes the mechanical properties of constituent $k$ as a function of the stretch $\lambda$, and this function changes with each time-step due to the evolution of constituent $k$.

A time increment from time $n \Delta t$ to time $(n+1) \Delta t$ is described below. The initial conditions, at $n=0$, are set according to Sect. 3.3 with $s_{0}=\hat{s}$. We also write Eq. (A.7) in discretized form for $n=0$, while letting $\lambda$ remain a continuous variable:

$$
\left.\frac{\partial}{\partial \lambda}\left(A^{k} \psi^{k}\right)\right|_{n=0}=A_{0}^{k} \frac{\hat{G}^{k}}{\lambda_{0}} d \Psi^{k}\left(\frac{\lambda}{\lambda_{0}}\right), \quad \forall k
$$

Integrals are approximated using the trapezoidal rule throughout. For a compact notation, we define a summation symbol used for the trapezoidal rule

$$
\sum_{i=m}^{n} a_{i}= \begin{cases}0 & , \quad m \geq n \\ \frac{1}{2} a_{m}+\sum_{i=m+1}^{n-1} a_{i}+\frac{1}{2} a_{n}, & \text { otherwise. }\end{cases}
$$

1. The equilibrium Eq. (20) and Eq. (29) give

$$
\left.\sum_{k} \frac{\partial}{\partial \lambda}\left(A^{k} \psi^{k}\right)\right|_{n}+A_{n}^{\mathrm{m}} \frac{\hat{G}^{\mathrm{m}}}{\langle\lambda\rangle_{n}^{\mathrm{m}}} S\left[C\left(\frac{\lambda_{n+1}}{\hat{\lambda}_{n+1}}\right)\right]-2 \pi R_{\mathrm{i}}^{2} p \lambda_{z} \lambda_{n+1}=0
$$

where the first term is a function of $\lambda=\lambda_{n+1}$. This Eq. (A.10) is solved for the stretch $\lambda_{n+1}$ using a numerical solver [f solve, GNU Octave, see Powell (1970)] with $\hat{\lambda}_{n+1}$ as the initial guess. If the solution is not unique, the solution closest to $\hat{\lambda}_{n+1}$ is chosen.

2. The stress measure $s_{n+1}$ is computed using Eq. (40b) with the recently computed value for $\lambda_{n+1}$ :

$$
s_{n+1}=\left.\sum_{k} \hat{\phi}^{k} \frac{1}{A_{n}^{k}} \lambda_{n+1} \frac{\partial}{\partial \lambda}\left(A^{k} \psi^{k}\right)\right|_{n}+\hat{\phi}^{\mathrm{m}} \frac{\hat{G}^{\mathrm{m}}}{\langle\lambda\rangle_{n}^{\mathrm{m}}} S\left[C\left(\frac{\lambda_{n+1}}{\hat{\lambda}_{n+1}}\right)\right] .
$$


3. For each constituent $k \in S_{\mathrm{i}}$, compute the newly produced materials according to Eqs. (A.3) and (44):

$$
\alpha_{n+1, n+1}^{k}=A_{n} v^{k} \hat{\phi}^{k}\left[1+\beta^{k}\left(\frac{\hat{\lambda}_{n+1}^{2}}{\lambda_{n+1}^{2}} \frac{s_{n+1}}{\hat{s}}-1\right)\right],
$$

where $A_{n}=\sum_{k} A_{n}^{k}$. Moreover, compute the amount of materials $\alpha_{n+1, j}^{k}, j=0,1, \ldots, n$ remaining from production at earlier times using Eq. (A.6).

4. Compute the effective area of constituents $k \in S_{\mathrm{i}}$ from Eq. (22) using the trapezoidal rule:

$$
A_{n+1}^{k}=A_{0}^{k} Q^{k}(n \Delta t+\Delta t)+\Delta t \sum_{j=0}^{n+1} * \alpha_{n+1, j}^{k}
$$

5. Express the weighted harmonic mean of the stretch using Eq. (30) and the trapezoidal rule:

$$
\langle\lambda\rangle_{n+1}^{\mathrm{m}}=A_{n+1}^{\mathrm{m}}\left[\frac{A_{0}^{\mathrm{m}}}{\lambda_{0}} Q^{\mathrm{m}}(n \Delta t+\Delta t)+\Delta t \sum_{j=0}^{n+1} \alpha_{n+1, j}^{\mathrm{m}} \frac{1}{\lambda_{j}}\right]^{-1} .
$$

6. Using the trapezoidal rule with Eq. (A.7) gives

$$
\begin{aligned}
\left.\frac{\partial}{\partial \lambda}\left(A^{k} \psi^{k}\right)\right|_{n+1}= & A_{0}^{k} Q^{k}(n \Delta t+\Delta t) \frac{\hat{G}^{k}}{\lambda_{0}} d \Psi^{k}\left(\frac{\lambda}{\lambda_{0}} \hat{G}^{k}\right)+ \\
& \Delta t \sum_{j=0}^{n+1} \alpha_{n+1, j}^{k} \frac{\hat{G}^{k}}{\lambda_{j}} d \Psi^{k}\left(\frac{\lambda}{\lambda_{j}} \hat{G}^{k}\right) .
\end{aligned}
$$

which is a function of $\lambda$ to be used for implicit calculation of $\lambda_{n+2}$ in the following time-step.

With these operations, all variables have been obtained for the time $(n+1) \Delta t$, and the new functions $\partial / \partial \lambda\left(A^{k} \psi^{k}\right)$ have been defined for use in the following time-step.

\section{Appendix A.3. Convergence for decreasing time-step}

Convergence is tested for $C^{\prime}=10$, for different values of $\beta^{\mathrm{c}}=\beta^{\mathrm{m}}=\{0.1,1,10\}$, and for different time-steps $v^{\mathrm{c}} \Delta t=$ $\{1 / 5,1 / 10,1 / 20,1 / 40,1 / 80,1 / 160\}$. The vessel is initially in a homeostatic state at a stretch $\lambda_{0}$ and total wall area $A_{0}=\sum_{k} A_{0}^{k}$ (Sect. 3.3). At $t=0$, the target stretch is increased to $\hat{\lambda}=$ $1.05 \lambda_{0}$. The development of $A=\sum_{k} A^{k}$ and $\lambda$ is integrated from $t=0$ to $t=100 / v^{\mathrm{c}}$ using the proposed numerical scheme (Fig. 2). Suppose a time-step $\Delta t$ yields an area $A_{i}^{\dagger}, i=0, \ldots, N$, while a time-step $2 \Delta t$ yields an area $A_{i}^{\ddagger}, i=0, \ldots, N / 2$. We define the nondimensional, root-mean-square of the difference between evolutions of $A$ due to halving of the time-step as

$$
\Delta E_{A}(\Delta t)=\sqrt{\frac{1}{t_{1} A_{0}^{2}} \sum_{i=0}^{N / 2}\left(A_{2 i}^{\dagger}-A_{i}^{\dagger}\right)^{2} 2 \Delta t}
$$

The corresponding root-mean-square $\Delta E_{\lambda}(\Delta t)$ for the evolutions of $\lambda$ due to halving of the time-step is analogously defined. We show that the solution converges as $\Delta t$ diminishes by plotting $\Delta E_{A}(\Delta t)$ and $\Delta E_{\lambda}(\Delta t)$ against the time-step in Fig. A.7.
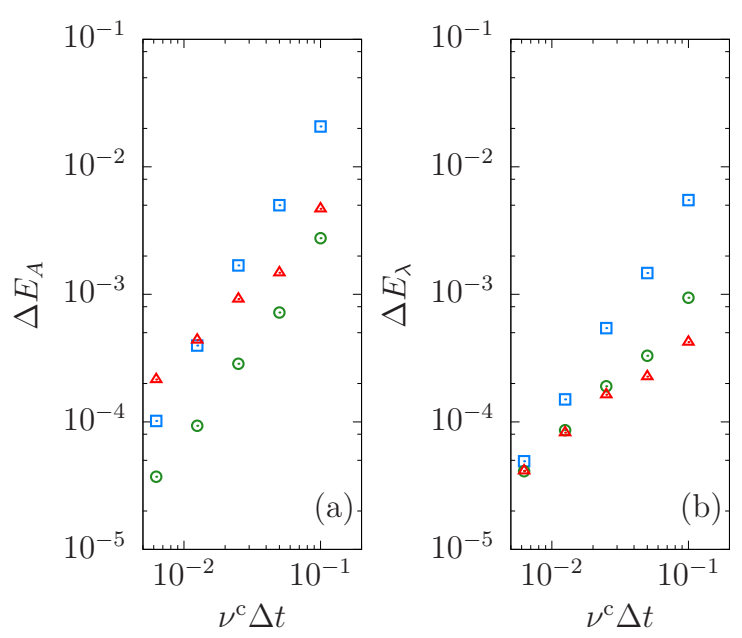

Figure A.7: Convergence of the solution for a $5 \%$ step increment in the target stretch with $\beta^{\mathrm{c}}=\beta^{\mathrm{m}}=0.1$ (squares), $\beta^{\mathrm{c}}=\beta^{\mathrm{m}}=1$ (circles), and $\beta^{\mathrm{c}}=\beta^{\mathrm{m}}=10$ (triangles). (a) Root-mean-square of the difference between area evolutions with time-steps $\Delta t$ and $2 \Delta t$, respectively. (b) Root-mean-square of the difference between stretch evolutions with time-steps $\Delta t$ and $2 \Delta t$, respectively.

Albinsson, S., Bhattachariya, A., Hellstrand, P., 2014. Stretch-dependent smooth muscle differentiation in the portal vein - role of actin polymerization, calcium signaling, and microRNA. Microcirculation 21, 230-238.

Baek, S., Rajagopal, K. R., Humphrey, J. D., 2006. A theoretical model of enlarging intracranial fusiform aneurysms. J. Biomech. Eng. 128 (1), 142149.

Baek, S., Valentín, A., Humphrey, J. D., 2007. Biomechanics of cerebral vasoplasm and its resolution: II. Constitutive relations and model simulations. Ann. Biomed. Eng. 35 (9), 1498-1509.

Boron, W. F., Boulpaep, E. L., 2008. Medical physiology. Saunders.

Brownlee, R. D., Langille, B. L., 1991. Arterial adaptations to altered blood flow. Can. J. Physiol. Pharm 69 (7), 978-983.

Canham, P., Talman, E. A., Finley, H. M., Dixon, J. G., 1991. Medial collagen organization in human arteries of the heart and brain by polarized light microscopy. Cell Tissue Res. 26, 121-134.

Dajnowiec, D., Langille, B. L., 2007. Arterial adaptations to chronic changes in haemodynamic function: Coupling vasomotor tone to structural remodelling. Clin. Sci. 113 (1), 15-23.

Finlay, H. M., McCullough, L., Canham, P. B., 1995. Three-dimensional collagen organization of human brain arteries at different transmural pressures. J. Vasc. Res. 32, 301-312.

Fridez, P., Makino, A., Kakoi, D., Miyazaki, H., Meister, J.-J., Hayashi, K., Stergiopulos, N., 2002. Adaptation of conduit artery vascular smooth muscle tone to induced hypertension. Ann. Biomed. Eng. 30 (7), 905-916.

Fujiwara, T., Uehara, Y., 1992. The cytoarchitecture of the medial layer in rat thoracic aorta: A scanning electron-microscopy study. Cell Tissue Res. 270, $165-172$.

Fung, Y. C., 1983. On the foundations of biomechanics. ASME J. Appl. Mech. 50, 1003-1009.

Gleason, R. L., Humphrey, J. D., 2004. A mixture model of arterial growth and remodeling in hypertension: Altered muscle tone and tissue turnover. J. Vasc. Res. 41 (4), 352-363.

Gleason, R. L., Wilson, E., Humphrey, J. D., 2007. Biaxial biomechanical adaptations of mouse carotid arteries cultured at altered axial extension. J. Biomech. 40 (4), 766-776.

Halayko, A. J., Solway, J., 2001. Plasticity in skeletal, cardiac, and smooth muscle cells. invited review: molecular mechanisms of phenotypic plasticity in smooth muscle cells. J. Appl. Physiol. 90, 358-368.

Holzapfel, G. A., Gasser, T. C., Ogden, R. W., 2000. A new constitutive framework for arterial wall mechanics and a comparative study of material models. J. Elasticity 61, 1-48.

Holzapfel, G. A., Ogden, R. W., 2010. Constitutive modeling of arteries. Proc. R. Soc. A 466, 1551-1596.

Horný, L., Adámek, A., Kulvajtová, M., 2016. A comparison of age-related 
changes in axial prestretch in human carotid arteries and in human abdominal aorta. Biomech. Model. Mechanobiol.Online.

Hu, J.-J., Baek, S., Humphrey, J. D., 2007a. Stress-strain behavior of the passive basilar artery in normotension and hypertension. J. Biomech. 40 (11), 2559-2563.

Hu, J.-J., Fossum, T. W., Miller, M. W., Xu, H., Liu, J.-C., Humphrey, J. D., 2007b. Biomechanics of the porcine basilar artery in hypertension. Ann. Biomed. Eng. 35 (1), 19-29.

Humphrey, J. D., 2002. Cardiovascular solid mechanics: Cells, tissues, and organs. Springer-Verlag, New York.

Humphrey, J. D., Rajagopal, K. R., 2002. A constrained mixture model for growth and remodeling of soft tissues. Math. Mod. Meth. Appl. S 12 (3), 407-430.

Humphrey, J. D., Wilson, E., 2003. A potential role of smooth muscle tone in early hypertension: a theoretical study. J. Biomech. 36, 1595-1601.

Kamenskiy, A. V., Pipinos, I. I., Dzenis, Y. A., Phillips, N. Y., Desyatova, A. S., Kitson, J., Bowen, R., MacTaggart, J. N., 2015. Effects of age on the physiological and mechanical characteristics of human femoropopliteal arteries. Acta Biomater. 11, 304-313.

Kamiya, A., Bukhari, R., Togawa, T., 1984. Adaptive regulation of wall shear stress optimizing vascular tree function. Bull. Math. Biol. 46 (1), 127-137.

Kubis, N., Checoury, A., Tedgui, A., Lévy, B. I., 2001. Adaptive common carotid arteries remodeling after unilateral internal carotid artery occlusion in adult patients. Cardiovasc. Res. 50 (3), 597-602.

Langille, B. L., Bendeck, M. P., Keeley, F. W., 1989. Adaptations of carotid arteries of young and mature rabbits to reduced carotid blood flow. Am. J. Physiol.-Heart C. 256 (4), H931-H939.

Langille, B. L., O’Donnell, F., 1986. Reductions in arterial diameter produced by chronic decreases in blood flow are endothelium-dependent. Science 231 (4736), 405-407.

Lefevre, M., Rucker, R. B., 1980. Aorta elastin turnover in normal and hypercholesterolemic Japanese quail. Biochim. Biophys. Acta 630, 519-529.

Lehman, R. M., Owens, G. K., Kassell, N. F., Hongo, K., 1991. Mechanism of enlargement of major cerebral collateral arteries in rabbits. Stroke 22 (4), 499-504.

Lindström, S. B., Satha, G., Klarbring, A., 2015. Extension of Murray's law including nonlinear mechanics of a composite artery wall. Biomech. Model. Mechan. 14 (1), 83-91.

Lodi, C. A., Ursino, M., 1999. Hemodynamic effect of cerebral vasospasm in humans: A modeling study. Ann. Biomed. Eng. 27, 257-273.

Matsumoto, T., Hayashi, K., 1996. Stress and strain distribution in hypertensive and normotensive rat aorta considering residual strain. J. Biomech. Eng. 118 (1), 62-71.

Murray, C. D., 1926. The physiological principle of minimum work. I. The vascular system and the cost of blood volume. Proc. Natl Acad. Sci. USA 12 (3), 207-214.

O’Connel, M. K., Murthy, S., Phan, S., Chengpei, X., Buchanan, J., Spilker, R., Dalman, R. L., Zarins, C. K., Denk, W., Taylor, C. A., 2008. The threedimensional micro- and nanostructure of the aortic medial lamellar unit measured using 3D confocal electron microscopy imaging. Matrix Biol. 27, $171-181$.

Ogden, R. W., 1997. Non-Linear Elastic Deformations.

Owens, G. K., Kumar, M. S., Wamhoff, B. R., 2001. Molecular regulation of vascular smooth muscle cell differentiation in development and disease. Physiol. Rev. 84, 767-801.

Pandolfi, A., Holzapfel, G. A., 2008. Three-dimensional modeling and computational analysis of the human cornea considering distributed collagen fibril orientations. J. Biomech. Eng. 130 (6), 061006.

Powell, M. J. D., 1970. A hybrid method for nonlinear equations. In: Rabinowitz, P. (Ed.), Numerical Methods for Nonlinear Algebraic Equations. Gordon and Breach.

Rachev, A., Hayashi, K., 1999. Theoretical study of the effects of vascular smooth muscle contraction on strain and stress distributions in arteries. Ann. Biomed. Eng. 27, 459-468.

Rhodin, J. A. C., 1979. Architecture of the vessel wall. In: Berne, R. M. (Ed.), Handbook of Pysiology. Section 2. American Physiological Society.

Rodbard, S., 1975. Vascular caliber. Cardiology 60, 4-49.

Satha, G., Lindström, S. B., Klarbring, A., 2014. A goal function approach to remodeling of arteries uncovers mechanisms for growth instability. Biomech. Model. Mechan. 13 (6), 1243-1259.

Schriefl, A. J., Zeindlinger, G., Pierce, D. M., Regitnig, P., Holzapfel, G. A.,
2012. Determination of the layer-specific distributed collagen fibre orientations in human thoracic and abdominal aortas and common iliac arteries. J. R. Soc. Interface 9 (71), 1275-1286.

Schulze-Bauer, C. A. J., Mörth, C., Holzapfel, G. A., 2003. Passive biaxial mechanical response of aged human iliac arteries. J. Biomech. Eng. 125, 395-406.

Skalak, R., Zargaryan, S., Jain, R., Netti, P., Hoger, A., 1996. Compatibility and the genesis of residual stress by volumetric growth. J. Math. Biol. 34, 889-914.

Taber, L. A., 1998. An optimization principle for vascular radius including the effects of smooth muscle tone. Biophys. J. 74, 109-114.

Takamizawa, K. K., Hayashi, K., 1987. Strain energy desity function and uniform strain hypothesis for arterial mechanics. J. Biomech. 20, 7-17.

Vaishnav, R. N., Vossoughi, J., 1983. Estimation of residual strains in aortic segments. In: Hall, C. W. (Ed.), Biomedical engineering II: Recent developments. Pergamon Press, New York.

Valentín, A., Cardamone, L., Baek, S., Humphrey, J. D., 2009. Complementary vasoactivity and matrix remodelling in arterial adaptations to altered flow and pressure. J. R. Soc. Interface 6 (32), 293-306.

Valentín, A., Humphrey, J. D., 2009a. Evaluation of fundamental hypotheses underlying constrained mixture models of arterial growth and remodelling. Philos. T. Roy. Soc. A 367 (1902), 3585-3606.

Valentín, A., Humphrey, J. D., 2009b. Parameter sensitivity study of a constrained mixture model of arterial growth and remodeling. J. Biomech. Eng. 131 (10), 101006.

Weizsäcker, H. W., Kampp, T. D., 1990. Passive elastic properties of the rat aorta. Biomed. Tech. 35, 224-234.

Zulliger, M. A., Kwak, N. T. M. R., Tsapikouni, T., Stergiopulos, N., 2002. Effects of longitudinal stretch on VSM tone and distensibility of muscular conduit arteries. Am. J. Physiol. Heart Circ. Physiol. 283, H2599-H2605.

Zulliger, M. A., Rachev, A., Stergiopulos, N., 2004. A constitutive formulation of arterial mechanics including vascular smooth muscle tone. Am. J. Physiol. Heart Circ. Physiol. 287, H1335-H1343. 